\title{
Absorption imaging and spectroscopy of ultracold neutral plasmas
}

\author{
T C Killian, Y C Chen, P Gupta, S Laha, Y N Martinez, P G Mickelson, \\ S B Nagel, A D Saenz and C E Simien
}

Department of Physics and Astronomy and Rice Quantum Institute, Rice University, Houston, TX, 77005, USA

Received 27 July 2004

Published 5 January 2005

Online at stacks.iop.org/JPhysB/38/S351

\begin{abstract}
Absorption imaging and spectroscopy can probe the dynamics of an ultracold neutral plasma during the first few microseconds after its creation. Quantitative analysis of the data, however, is complicated by the inhomogeneous density distribution, expansion of the plasma and possible lack of global thermal equilibrium for the ions. In this paper, we describe methods for addressing these issues. Using simple assumptions about the underlying temperature distribution and ion motion, the Doppler-broadened absorption spectrum obtained from plasma images can be related to the average temperature in the plasma.
\end{abstract}

(Some figures in this article are in colour only in the electronic version)

\section{Introduction}

Ultracold neutral plasmas [1], formed by photoionizing laser-cooled atoms near the ionization threshold, stretch the boundaries of traditional neutral plasma physics. The electron temperature in these plasmas is from $1-1000 \mathrm{~K}$ and the ion temperature is around $1 \mathrm{~K}$. The density can be as high as $10^{10} \mathrm{~cm}^{-3}$. Fundamental interest in these systems stems from the possibility of creating strongly coupled plasmas [2], but collective modes [3], recombination [4] and thermalization [5] have also been studied.

Charged particle detection techniques have traditionally been used for these experiments. However, optical absorption imaging and spectroscopy, demonstrated in [5] using the $\mathrm{Sr}^{+}$ ${ }^{2} \mathrm{~S}_{1 / 2} \rightarrow{ }^{2} \mathrm{P}_{1 / 2}$ transition in a strontium plasma, opens many new possibilities. Images depict the density profile of the plasma, and the Doppler-broadened absorption spectrum measures the ion velocity distribution. Both can probe ion dynamics with $50 \mathrm{~ns}$ resolution.

Qualitative interpretation of the images and spectra is straightforward, but quantitative analysis is complicated by the inhomogeneous density distribution of the plasma, plasma expansion, and the possible lack of global thermal equilibrium for the ions. In order to address these effects, we are forced to make some simple assumptions about the form of the 

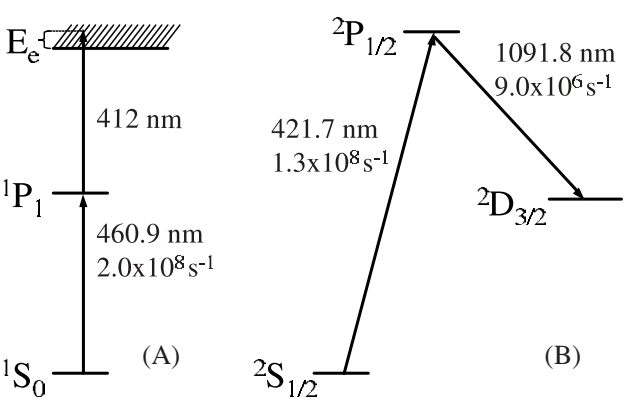

Figure 1. Strontium atomic and ionic energy levels involved in the experiment, with decay rates. (A) Neutral atoms are laser cooled and trapped in a magneto-optical trap (MOT) operating on the ${ }^{1} \mathrm{~S}_{0}-{ }^{1} \mathrm{P}_{1}$ transition at $460.9 \mathrm{~nm}$, as described in [6]. Atoms excited to the ${ }^{1} \mathrm{P}_{1}$ level by the MOT lasers are ionized by photons from a laser at $\sim 412 \mathrm{~nm}$. (B) Ions are imaged using the ${ }^{2} \mathrm{~S}_{1 / 2}-{ }^{2} \mathrm{P}_{1 / 2}$ transition at $421.7 \mathrm{~nm} .{ }^{2} \mathrm{P}_{1 / 2}$ ions decay to the ${ }^{2} \mathrm{D}_{3 / 2}$ state $7 \%$ of the time, after which they cease to interact with the imaging beam. This does not complicate the experiment because ions typically scatter fewer than one photon during the time the imaging beam is on.

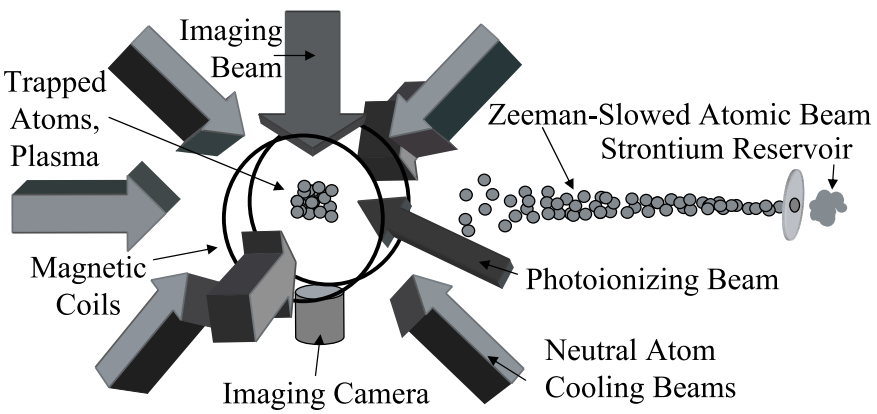

Figure 2. From [5]. Experimental schematic for strontium plasma experiments. The MOT for neutral atoms consists of a pair of anti-Helmholtz magnetic coils and six laser-cooling beams. Atoms from a Zeeman-slowed atomic beam enter the MOT region and are trapped. ${ }^{1} \mathrm{P}_{1}$ atoms are ionized by the photoionizing laser. The imaging beam passes through the plasma and falls on a CCD camera.

ion temperature distribution and expansion of the plasma. In this paper, we motivate these assumptions and describe our methods for quantitatively analysing the data.

Section 2 provides an overview of the plasma creation and absorption imaging technique. Section 3 describes the dynamics of the ions during the first few microseconds after photoionization. This provides the basis for understanding section 4, which explains how the absorption spectrum is extracted from the images and how it relates to the temperature distribution.

\section{Experimental overview}

The production of a strontium plasma starts with atoms that are cooled and confined in a magneto-optical trap (MOT) (figures 1 and 2). This aspect of the experiment was described in [6]. The neutral atom cloud is characterized by a temperature of a few $\mathrm{mK}$ and a density distribution given by $n(r)=n_{0} \exp \left(-r^{2} / 2 \sigma^{2}\right)$, with $\sigma \approx 0.6 \mathrm{~mm}$ and $n_{0} \approx 6 \times 10^{10} \mathrm{~cm}^{-3}$. The number of trapped atoms is typically $2 \times 10^{8}$. These parameters can be adjusted. In particular, turning off the trap and allowing the cloud to expand yields larger samples with lower densities. 


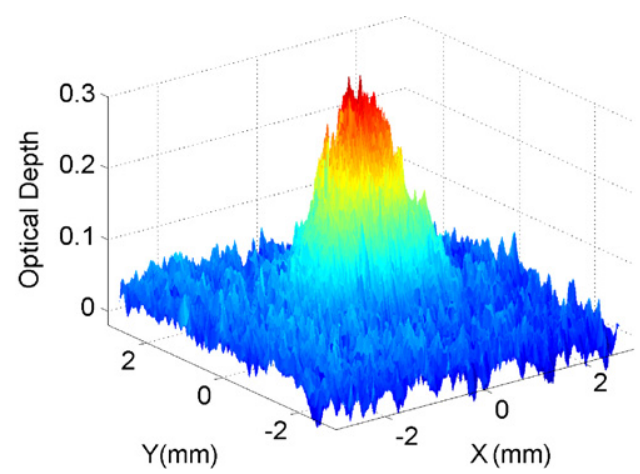

Figure 3. Optical depth of an ultracold neutral plasma. The delay between the formation of the plasma and image exposure is $85 \mathrm{~ns}$. The plasma contains $7 \times 10^{7}$ ions and the initial peak ion density is $n_{0 i}=2 \times 10^{10} \mathrm{~cm}^{-3}$. Resolution is about $65 \mu \mathrm{m}$, limited by pixel averaging to improve the signal-to-noise ratio. The intensity of the probe beam is approximately $200 \mu \mathrm{W} \mathrm{cm} \mathrm{cm}^{-2}$, which is much less than the saturation intensity of the transition $\left(38 \mathrm{~mW} \mathrm{~cm}^{-2}\right)$. The probe beam diameter is about 10 times the diameter of the plasma.

To form the plasma, the MOT magnets are turned off and atoms are ionized with photons from the cooling laser and from a 10 ns pulsed dye laser whose wavelength is tuned just above the ionization continuum (figure 1). Up to $30 \%$ of the neutral atoms are ionized, producing plasmas with a peak electron and ion density as high as $n_{0 e} \approx n_{0 i} \approx 2 \times 10^{10} \mathrm{~cm}^{-3}$. The density profiles, $n_{e}(r) \approx n_{i}(r)$, follow the Gaussian shape of the neutral atom cloud.

Because of the small electron-ion mass ratio, the electrons have an initial kinetic energy approximately equal to the difference between the photon energy and the ionization potential, typically between 1 and $1000 \mathrm{~K}$. The initial kinetic energy for the ions is close to the kinetic energy of neutral atoms in the MOT. As we will discuss below, the resulting non-equilibrium plasma evolves rapidly.

To record an absorption image of the plasma, a collimated laser beam, tuned near resonance with the principle transition in the ions, illuminates the plasma and falls on an image intensified CCD camera. Ions scatter photons out of the laser beam and create a shadow that is recorded by the CCD camera. The optical depth $(O D)$ is defined in terms of the image intensity without ( $\left.I_{\text {background }}\right)$ and with $\left(I_{\text {plasma }}\right)$ the plasma present,

$$
O D(x, y)=\ln \left(I_{\text {background }}(x, y) / I_{\text {plasma }}(x, y)\right) .
$$

Figure 3 shows a typical absorption image.

To study the time evolution of the plasma, we vary the delay between the formation of the plasma and image exposure $\left(t_{\text {delay }}\right)$ with $10 \mathrm{~ns}$ accuracy. The minimum camera exposure gate width is $50 \mathrm{~ns}$. For the shortest exposure times, which we use for the best time resolution at very short $t_{\text {delay }}$, we typically average about 60 ionizing laser shots. For longer delay times we use longer image exposure times, and decrease the number of accumulations to keep the total number of photons detected approximately constant. The repetition rate for ionization and image recording is about 5 times per second.

\section{Ion dynamics}

In order to understand the details of the image analysis, it is necessary to understand the dynamics of the plasma. The imaging probe is most sensitive to the ion dynamics, so we will 
concentrate on this topic. The behaviour of electrons was studied experimentally in $[1,3,4]$ and theoretically in [7-9].

Ions are created with very little kinetic energy, but their initial spatially-uncorrelated state possesses significant Coulomb potential energy compared to the regular lattice that represents the ground state of the system [10,11]. As ions equilibrate and correlations develop, the kinetic energy increases. This process is called disorder-induced heating, or correlation heating, and it has been discussed in many theoretical papers. Early interest was generated by non-equilibrium plasmas created by fast-pulse laser irradiation of solid targets, for example [12-15], and the problem has been revisited in the context of ultracold neutral plasmas [7, 8, 16, 17].

Qualitatively, one expects the ion temperature after equilibration to be on the order of the Coulomb interaction energy between neighbouring ions. A quantitative analysis [16], assuming complete initial disorder and incorporating the screening effects of the electrons, predicts an ion temperature of

$$
T_{i}=\frac{2}{3} \frac{e^{2}}{4 \pi \varepsilon_{0} a k_{\mathrm{B}}}\left|\tilde{U}+\frac{\kappa}{2}\right| .
$$

Here, $\kappa=a / \lambda_{D}$ where $\lambda_{D}=\left(\varepsilon_{0} k_{\mathrm{B}} T_{e} / n_{e} e^{2}\right)^{1 / 2}$ is the Debye length. The quantity $\tilde{U} \equiv \frac{U}{N_{i} e^{2} / 4 \pi \varepsilon_{0} a}$ is the excess potential energy per particle in units of $e^{2} / 4 \pi \varepsilon_{0} a$, where $a=\left(4 \pi n_{i} / 3\right)^{-1 / 3}$ is the Wigner-Seitz radius, or interparticle distance. $N_{i}$ is the number of ions. $\tilde{U}$ has been studied with molecular dynamics simulations [18] for a homogeneous system of particles interacting through a Yukawa potential, $\phi(r)=\frac{e^{2}}{4 \pi \varepsilon_{0} r} \exp \left(-r / \lambda_{D}\right)$, which describes ions in the background of weakly coupled electrons ${ }^{1}$.

For typical strontium plasmas discussed here, $\kappa \approx 0.1-1$, and $\lambda_{D} \approx 2-8 \mu \mathrm{m}$. $\tilde{U}$ ranges from -0.6 to -0.8 , so $T_{i}$ is close to $e^{2} / 4 \pi \varepsilon_{0} a k_{\mathrm{B}}$ as expected. $\kappa$ is related to the Coulomb coupling parameter for electrons, $\Gamma_{e}$, through $\kappa=\sqrt{3 \Gamma_{e}}$. A system is strongly coupled when $\Gamma>1$ [2]. $\Gamma_{e} \approx 0.1-0.5$ for the systems studied here, so the electrons are not strongly coupled. This avoids excessive complications that arise when $\Gamma_{e}$ approaches or initially exceeds unity, such as screening of the ion interaction [7], and rapid collisional recombination and heating of the electrons [7-9, 19], although we do see some signs of these effects, even in this regime. The ions typically equilibrate with $T_{i} \approx 1 \mathrm{~K}$, which gives $\Gamma_{i} \approx 3$, so the ions are strongly coupled.

The time scale for disorder-induced heating is the inverse of the ion plasma oscillation frequency, $1 / \omega_{p i}=\sqrt{m_{i} \varepsilon_{0} / n_{i} e^{2}}$, which is of the order of $100 \mathrm{~ns}$. Physically, this is the time for an ion to move one interparticle spacing when accelerated by a typical Coulomb force of $e^{2} / 4 \pi \varepsilon_{0} a^{2}$. This time scale is also evident in molecular dynamics simulations of ion-ion thermalization [7, 8, 12-17].

It is interesting to note that under usual conditions in weakly interacting plasmas or even atomic gases, the time scale for relaxation of the two-particle distribution function, which describes correlations, is much faster than the collision time that governs the relaxation of the one-particle distribution function to the Maxwell-Boltzmann form. This is known as Bogoliubov's hypothesis [20]. For strongly coupled plasmas, however, these time scales both become equal to the inverse of the plasma oscillation frequency [13].

As the two-particle distribution function equilibrates, the kinetic energy of the ions exhibits strongly damped oscillations at twice the ion plasma oscillation frequency. Intuitively, this can be understood as the oscillation of each ion in its local potential energy well. It is questionable whether this should be called an ion plasma oscillation or not because there is probably

1 As the number of electrons per Debye sphere $\left(\kappa^{-3}\right)$ approaches unity, the Yukawa interaction ceases to accurately describe ion-ion interactions. For strontium plasmas studied here, this situation only occurs for the highest $n_{e}$ and lowest $T_{e}$. It will be interesting to test equation (2) for these conditions. 
no collective or long range coherence to the motion. This behaviour has been observed in molecular dynamics simulations of equilibrating strongly coupled systems $[13,15,17]$. The damping time for the oscillations is approximately $\pi / \omega_{p i}$ for $\Gamma \geqslant 5$. Averaging over the entire density distribution, as we do in the analysis described here, obscures the oscillations. A different approach, which resolves regions of different density in the plasma, is required to clearly observe the oscillations, and this phenomenon will not be discussed further in this paper.

For $t_{\text {delay }}>\pi / \omega_{p i}$, the ions have equilibrated and the thermal energy of the electrons begins to dominate the evolution of the plasma. Electrons contained in the potential created by the ions exert a pressure on the ions that causes an outward radial acceleration. This was studied experimentally in [3] and theoretically by a variety of means in [9]. The experiments measured the final velocity that the ions acquired, which was approximately $v_{\text {terminal }} \approx \sqrt{E_{e} / m_{i}}$. With the imaging probe, we now observe the expansion dynamics at much earlier times during the acceleration phase.

As discussed in [3, 5], a hydrodynamic model, which describes the plasma on length scales larger than $\lambda_{D}$, shows that the pressure of the electron gas drives the expansion through an average force per ion of

$$
\bar{F}=\frac{-\bar{\nabla}\left(n_{e}(r) k_{\mathrm{B}} T_{e}\right)}{n_{i}(r)} \approx \hat{r} \frac{r k_{\mathrm{B}} T_{e}}{\sigma_{i}^{2}},
$$

where the ion and electron density distributions are $n_{e}(r) \approx n_{i}(r)=n_{0 i} \exp \left(-r^{2} / 2 \sigma_{i}^{2}\right)$. We assume thermal equilibrium for the electrons throughout the cloud [9].

The force leads to an average radial expansion velocity for the ions,

$$
\bar{v}\left(r, t_{\text {delay }}\right)=\hat{r} \frac{r k_{\mathrm{B}} T_{e}}{m_{i} \sigma_{i}^{2}} t_{\text {delay }} .
$$

The velocity is correlated with position and increases linearly with time. This does not represent an increase in the random thermal velocity spread or temperature of the ions. Due to the large mass difference, thermalization of ions and electrons [7] is slow and occurs on a millisecond time scale.

Equation (4) for the average ion velocity assumes a constant electron temperature. Actually, as the plasma expands, electrons will cool. This can be thought of in terms of energy conservation or adiabatic expansion. It is possible to describe the expansion with a Vlasov equation that includes the changing electron temperature. For an initial Gaussian density distribution, the equations can be solved analytically and the expansion preserves the Gaussian shape with a $1 / \sqrt{e}$ density radius given by $\sigma_{i}^{2}(t) \approx \sigma_{i}^{2}(0)+\left[k_{\mathrm{B}} T_{e}(0) / m_{i}\right] t^{2}$ $[9,21,22]$. The experiments involving absorption imaging of the plasma, however, have concentrated on the first few microseconds of the expansion when the plasma size and electron temperature have not changed significantly. Thus we can safely use equation (4). The effects of the expansion are evident in the radial velocity that manifests itself in Doppler broadening of the ion absorption spectrum.

\section{Doppler-broadened spectrum}

To obtain quantitative information from the plasma images, we relate the $O D$ (equation (1)) to underlying physical parameters. Following Beer's law, the $O D$ for a laser propagating along the $z$ axis is

$$
O D(x, y)=\int \mathrm{d} z n_{i}(x, y, z) \alpha\left[v, T_{i}(r)\right]
$$




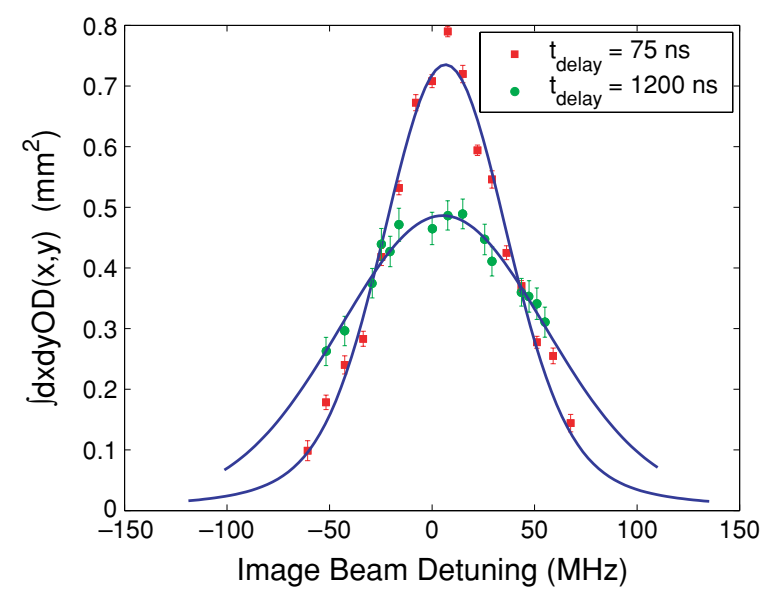

Figure 4. Absorption spectra of ultracold neutral plasmas. We plot the integral of the optical depth (equation (6)). The frequency is with respect to a Doppler-free absorption feature in a strontium discharge cell. Both spectra correspond to $T_{e}=56 \mathrm{~K}$ and an initial peak plasma density of $n_{0 i}=2 \times 10^{10} \mathrm{~cm}^{-3}$. Data are fit with Voigt profiles, and the increase in linewidth for longer $t_{\text {delay }}$ is clear.

where $n_{i}(x, y, z)$ is the ion density, and $\alpha\left[v, T_{i}(r)\right]$ is the ion absorption cross section at the image beam frequency, $v$. The absorption cross section is a function of temperature due to Doppler broadening, and since we expect the temperature to vary with density, we allow $\alpha$ to vary with position. If we now integrate over $x$ and $y$, or, in reality, sum over the image pixels multiplied by the pixel area, we get the spectrum

$$
S(v) \equiv \int \mathrm{d} x \mathrm{~d} y O D(x, y)=\int \mathrm{d}^{3} r n_{i}(r) \alpha\left[v, T_{i}(r)\right],
$$

as a function of the image laser detuning ${ }^{2}$. As we vary the detuning, we obtain absorption spectra as shown in figure 4 . The rest of the paper will deal with the relationship between spectra such as these and the underlying temperature distributions of the ions.

The absorption cross section for ions in a region described by a temperature $T_{i}$, is given by the Voigt profile

$$
\alpha\left(\nu, T_{i}\right)=\int \mathrm{d} s \frac{3^{*} \lambda^{2}}{2 \pi} \frac{1}{1+4\left(\frac{\nu-s}{\gamma_{\text {eff }} / 2 \pi}\right)^{2}} \frac{1}{\sqrt{2 \pi} \sigma_{D}\left(T_{i}\right)} \mathrm{e}^{-\left(s-\nu_{0}\right)^{2} / 2 \sigma_{D}\left(T_{i}\right)^{2}},
$$

where $\sigma_{D}\left(T_{i}\right)=\sqrt{k_{\mathrm{B}} T_{i} / m_{i}} / \lambda$ is the Doppler width, and $\gamma_{\text {eff }}=\gamma_{0}+\gamma_{\text {laser }}$ is the effective Lorentzian linewidth due to the natural linewidth of the transition, $\gamma_{0}=2 \pi \times 22 \times 10^{6} \mathrm{rad} \mathrm{s}^{-1}$, and the laser linewidth, $\gamma_{\text {laser }}=2 \pi \times(5 \pm 2) \times 10^{6} \mathrm{rad} \mathrm{s}^{-1}$. The centre frequency of the transition is $\nu_{0}=c / \lambda$, where $\lambda=422 \mathrm{~nm}$. The 'three-star' symbol, $3^{*}=1$, accounts for the equal distribution of ions in the doubly degenerate ground state and the linear polarization of the imaging light [23].

Extracting the ion temperature from the spectrum is complicated by the fact that we do not expect to have global thermal equilibrium of the ions during the first microsecond after

2 We can also fit $O D(x, y)$ to a two-dimensional Gaussian, as described in [5], and identify $\int \mathrm{d} x \mathrm{~d} y O D(x, y) \approx$ $2 \pi \sigma_{i x} \sigma_{i y} O D_{\max }$, where $\sigma_{i x}$ and $\sigma_{i y}$ are the transverse sizes of the absorption profile, and $O D_{\max }$ is the peak optical depth. This sometimes has signal-to-noise ratio advantages over integrating the entire image, but both approaches should give the same result. 


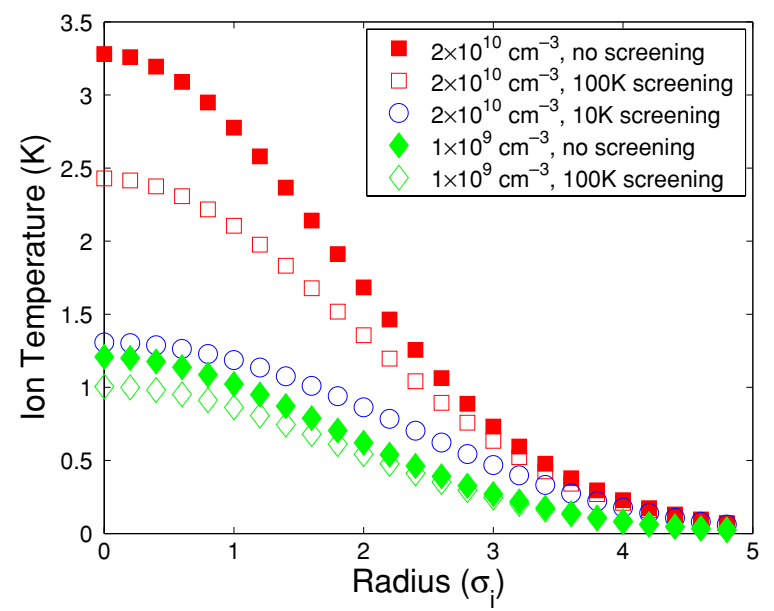

Figure 5. Ion temperature profiles. Peak densities $\left(n_{0, i} \approx n_{0, e}\right)$ and electron temperatures are given in the legend. Screening refers to the iterative calculation of temperature using equation (2). No screening refers to equation (8), which neglects electron screening, and is thus independent of electron temperature. In the limit of low density and high electron temperature, both expressions give the same result.

plasma formation. Global thermal equilibrium will occur on a hydrodynamic time scale, $\sigma_{i} / v$, which is on the order of $10 \mathrm{~ms}$, where $v$ is the ion acoustic wave velocity ${ }^{3}$.

We need a method to relate the Doppler broadening of the spectrum to a characteristic temperature. We will show that for reasonable models of the ion temperature distribution, the temperature extracted from a fit of a Voigt profile to the spectrum yields a temperature that is a close approximation of the average ion temperature in the plasma. More thorough checks of this assignment, such as with molecular dynamics simulations, would be valuable, but this serves as a useful working definition for discussing the data.

For times longer than $\pi / \omega_{p i}$, but short compared to hydrodynamic times, we expect local thermal equilibrium at a temperature approximately given by equation (2). Under this model, the temperature varies across the plasma because the interparticle spacing, $a$, varies with density. Given $T_{e}$ and $n_{i}$, it is possible to use equation (2) in an iterative recipe to find the local ion temperature. This procedure utilizes an expression [24] for the excess potential energy $\tilde{U}$ in terms of $\Gamma_{i}$ and $\kappa$ that is valid for $\kappa<5$. This yields ion temperature distributions as shown in figure 5 .

To simplify the discussion, we first neglect electron screening. This accurately describes the plasma for $\kappa \ll 1$ (high $T_{e}$ and low $n_{e}$ ). To a good approximation, we can then say

$$
T_{i}(r)=C \frac{e^{2}}{4 \pi \varepsilon_{0} a(r) k_{\mathrm{B}}}=T_{i, \max } \mathrm{e}^{-r^{2} / 6 \sigma_{i}^{2}},
$$

where $T_{i, \max }$ is the temperature corresponding to the peak density at $r=0$. Molecular dynamics simulations of ultracold plasmas that neglect electron screening display this thermal distribution when the ions have equilibrated locally $\left(t_{\text {delay }} \gtrsim \pi / \omega_{p i}\right)$ [25]. $C=0.45$ is a constant determined by numerical evaluation of the parameters in equation (2) for small $\kappa{ }^{4}$

3 In principle, it is possible to obtain spectra from small regions of the cloud, so as to examine the local dynamics and avoid averaging over the plasma. Developing this capability will be the subject of future studies.

4 As $\kappa$ increases, equation (8) still describes the ion temperature if $C$ decreases and becomes a function of position in the plasma, as shown in figure 5. 


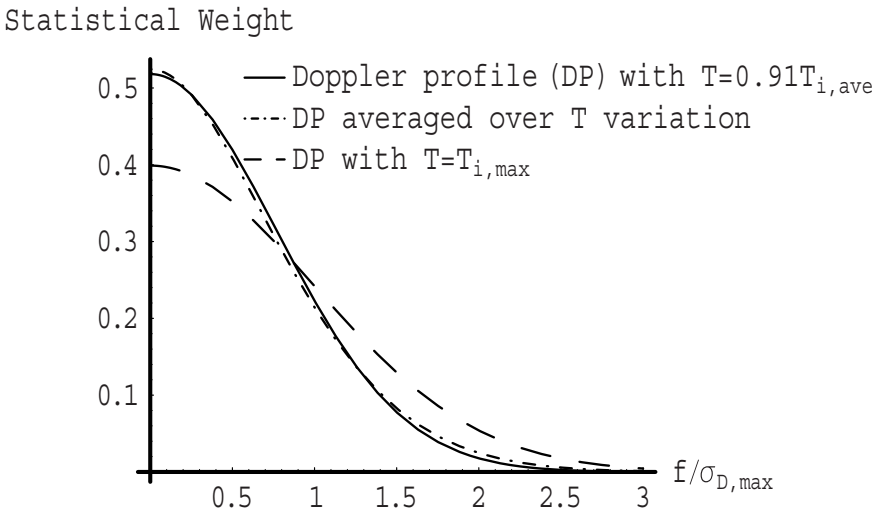

Figure 6. Assuming $T_{i}(r)$ is given by equation (8), the Doppler profile averaged over the density distribution can be replaced by a Doppler profile characterized by a single uniform temperature, $T=0.59 T_{i, \max }=0.91 T_{i, \text { ave }}$. The statistical weight is proportional to the probability of finding ions with that Doppler shift. The horizontal axis is the Doppler shift away from the unperturbed resonance in units of $\sigma_{D \text {, max }}$.

The average ion temperature, assuming equation (8), is

$$
T_{i, \text { ave }}=\frac{1}{N_{i}} \int \mathrm{d}^{3} r n_{i}(r) T_{i}(r)=T_{i, \max } \frac{3 \sqrt{3}}{8} .
$$

Combining the expressions for the integral over the optical depth (equation (6)), and the expression for the absorption cross section (equation (7)) yields

$$
S(v)=\int \mathrm{d}^{3} r n_{i}(r) \int \mathrm{d} s \frac{3^{*} \lambda^{2}}{2 \pi} \frac{1}{1+4\left(\frac{v-s}{\gamma_{\text {eff }} / 2 \pi}\right)^{2}} \frac{1}{\sqrt{2 \pi} \sigma_{D}\left[T_{i}(r)\right]} \mathrm{e}^{-\left(s-\nu_{0}\right)^{2} / 2 \sigma_{D}\left[T_{i}(r)\right]^{2}} .
$$

If we insert equation (8) for $T_{i}(r)$, we find

$$
\begin{aligned}
S(v)=\int \mathrm{d} s & \frac{3^{*} \lambda^{2}}{2 \pi} \frac{1}{1+4\left(\frac{\nu-s}{\gamma_{\text {eff }} / 2 \pi}\right)^{2}} \int \mathrm{d}^{3} r n_{0 i} \mathrm{e}^{-r^{2} / 2 \sigma_{i}^{2}} \\
& \times \frac{1}{\sqrt{2 \pi} \sigma_{D, \text { max }}} \mathrm{e}^{r^{2} / 12 \sigma_{i}^{2}} \exp \left[-\mathrm{e}^{r^{2} / 6 \sigma_{i}^{2}}\left(s-v_{0}\right)^{2} / 2 \sigma_{D, \text { max }}^{2}\right] .
\end{aligned}
$$

Here $\sigma_{D, \max }$ is the Doppler width corresponding to $T_{i, \max }$. As shown in figure 6 , the average over the density distribution of the Doppler factor can be replaced by a single Doppler distribution with temperature $T=0.59 T_{i, \max }=0.91 T_{i, \text { ave }}$.

$$
\begin{gathered}
\int \mathrm{d}^{3} r n_{0 i} \mathrm{e}^{-r^{2} / 2 \sigma_{i}^{2}} \frac{1}{\sqrt{2 \pi} \sigma_{D, \max }} \mathrm{e}^{r^{2} / 12 \sigma_{i}^{2}} \exp \left[-\mathrm{e}^{r^{2} / 6 \sigma_{i}^{2}}\left(s-v_{0}\right)^{2} / 2 \sigma_{D, \max }^{2}\right] \\
\approx \frac{N_{i}}{\sqrt{2 \pi} \tilde{\sigma}_{D}} \exp \left[-\left(s-v_{0}\right)^{2} / 2 \tilde{\sigma}_{D}^{2}\right],
\end{gathered}
$$

where $\tilde{\sigma}_{D}=\sqrt{0.91 k_{\mathrm{B}} T_{i, \text { ave }} / m_{i}} / \lambda$. So if the velocity distribution of the ions is characterized by local thermal equilibrium of the form in equation (8), fitting the spectrum, $S(v)$, to a Voigt profile yields a good approximate measure of the quantity $T_{i, \text { ave }}$.

If we want to include the effects of electron screening, we can follow exactly the same procedure, except in our model of the spectrum (equation (10)) we use the iterative recipe for ion temperature using equation (2), instead of the simple equation (8). This slightly 
more complicated numerical analysis shows that if equation (2) describes the temperature distribution, then the temperature extracted from the Voigt profile is between 0.91 and 0.95 of $T_{i, \text { ave }}$. In this case, the average ion temperature is found by averaging equation (2) over the density distribution.

If we have global thermal equilibrium, which we expect for long $t_{\text {delay }}$, then the temperature is uniform, and, by definition, the Voigt profile will yield the average temperature.

The expansion of the plasma, equation (4), also contributes to the velocity distribution. So we must expand our model. Fortunately, the expansion is well understood $[3,9,22]$ and determined by the parameters $T_{e}$ and $\sigma_{i}$. Independent analysis of the images determines $\sigma_{i}$. $T_{e}$ is approximately equal to $2 E_{e} / 3 k_{\mathrm{B}}$, where $E_{e}$ is the detuning of the photoionizing laser above resonance [1], although for high density and low electron temperature, we can expect a small increase of $T_{e}$ above $2 E_{e} / 3 k_{\mathrm{B}}$ due to electron heating from three-body recombination [9], disorder-induced heating [7] and continuum lowering [8].

We will first treat the expansion using the simple model of ion temperature, equation (8). The expansion velocity profile gives rise to an average Doppler shift of the resonant frequency that varies with position,

$$
\begin{aligned}
\delta v=v_{z} / \lambda & =\frac{r k_{\mathrm{B}} T_{e} t_{\text {delay }}}{m_{i} \sigma_{i}^{2} \lambda} \cos \theta \\
& =\sigma_{D, \text { ave }} \frac{r}{\sigma_{i}} \frac{t_{\text {delay }}}{t_{\text {exp }}} \cos \theta,
\end{aligned}
$$

where $r \cos \theta$ is the displacement from the centre of the cloud along the direction of laser propagation. We have introduced a characteristic time for the expansion,

$$
t_{\exp }=\sigma_{i} \sqrt{\frac{m_{i}}{k_{\mathrm{B}} T_{e}}} \sqrt{\frac{T_{i, \text { ave }}}{T_{e}}},
$$

which is the time at which the Doppler shift due to expansion, at $r \cos \theta=\sigma_{i}$, equals $\sigma_{D \text {,ave }}$. In other words, this is the time at which the Doppler broadening due to expansion becomes comparable to the thermal Doppler broadening. For typical plasma conditions, such as $T_{e}=$ $30 \mathrm{~K}, T_{i, \text { ave }}=1 \mathrm{~K}$, and $\sigma_{i}=1 \mathrm{~mm}$, equation (14) yields $t_{\exp }=3.4 \mu \mathrm{s}$.

We include the position-dependent Doppler shift in the exponent of the Gaussian describing the Doppler broadening in the Voigt convolution (equation (11)), which becomes

$$
\begin{aligned}
& \int \mathrm{d}^{3} r n_{0 i} \mathrm{e}^{-r^{2} / 2 \sigma_{i}^{2}} \frac{1}{\sqrt{2 \pi} \sigma_{D, \max }} \mathrm{e}^{-r^{2} / 12 \sigma_{i}^{2}} \\
& \quad \times \exp \left[-\mathrm{e}^{r^{2} / 6 \sigma_{i}^{2}}\left(s-v_{0}-\sigma_{D, \text { ave }} \frac{r}{\sigma_{i}} \frac{t_{\text {delay }}}{t_{\exp }} \cos \theta\right)^{2} / 2 \sigma_{D, \text { max }}^{2}\right] \\
& \approx \frac{N_{i}}{\sqrt{2 \pi} \sigma_{D}\left(T_{i, \text { eff }}\right)} \exp \left[-\frac{\left(s-v_{0}\right)^{2}}{2 \sigma_{D}^{2}\left(T_{i, \text { eff }}\right)}\right] .
\end{aligned}
$$

In the second line of equation (15), we have given the Doppler profile, corresponding to a temperature of

$$
T_{i, \text { eff }}=C T_{i, \text { ave }}\left[1+\frac{1}{C}\left(\frac{t_{\text {delay }}}{t_{\text {exp }}}\right)^{2}\right]
$$

that approximately equals the original full integral, including variation in temperature across the plasma and effects of expansion. Here, $C=(0.95 \pm 0.05)$. Figure 7 shows that the agreement is good over the entire time scale of the expansion. 


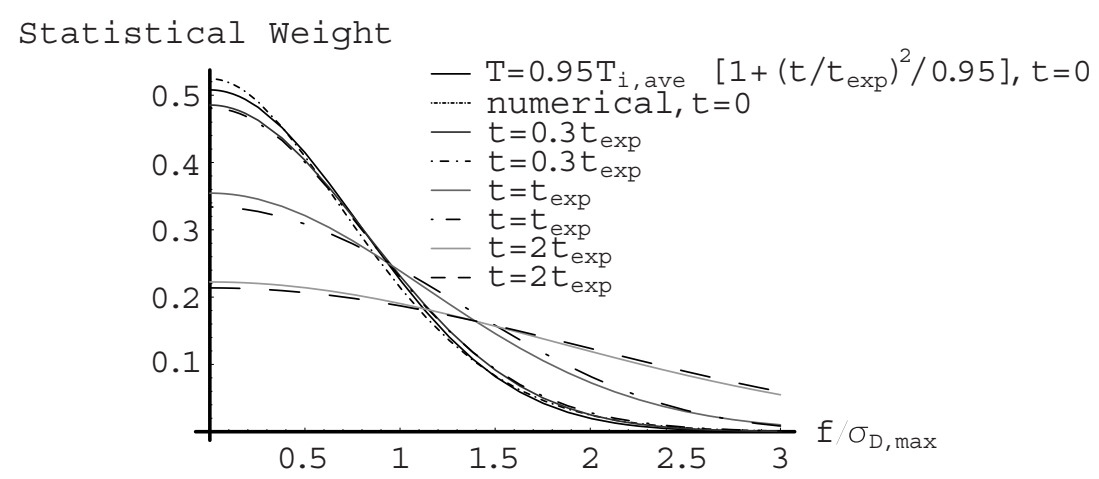

Figure 7. Doppler profile averaged over the density distribution as in figure 6, but now including the effects of expansion. The dashed or dashed-dotted numerical expressions are full integrals over volume shown in equation (15). The solid curves are the Gaussians for Doppler broadening arising from a single temperature $T=0.95 T_{i \text {,ave }}\left[1+(1 / 0.95)\left(t / t_{\text {exp }}\right)^{2}\right]$, where $t_{\text {exp }}$ is the characteristic time scale for the expansion described in the text. At all times, the single Doppler profile is a good approximation to the full integral.

If we include screening effects and use equation (2) to find the temperature, then numerical simulation shows that the extracted temperature is also given by equation (16), with $C$ ranging from 0.91 to 0.95 . If the plasma has reached global thermal equilibrium, then it can be shown analytically that $T=T_{i \text {,ave }}\left[1+\left(t_{\text {delay }} / t_{\text {exp }}\right)^{2}\right]$ exactly. This suggests that when fitting a Voigt profile to the spectrum derived from the integral over the optical depth,

$$
S(\nu)=N_{i} \int \mathrm{d} s \frac{3^{*} \lambda^{2}}{2 \pi} \frac{1}{1+4\left(\frac{v-s}{\gamma_{\text {eff }} / 2 \pi}\right)^{2}} \frac{1}{\sqrt{2 \pi} \sigma_{D}\left(T_{i, \text { eff }}\right)} \mathrm{e}^{-\left(s-\nu_{0}\right)^{2} / 2 \sigma_{D}\left(T_{i, \text { eff }}\right)^{2}},
$$

the extracted effective temperature can be related to the average temperature in the plasma through equation (16). One can show analytically that equation (16) has the correct form in the limit when all broadening is negligible except the Doppler broadening due to expansion.

Our motivation of equation (16) relies on certain models of the ion temperature. They are reasonable approximations to the distributions seen in molecular dynamics simulations except at short times, $t<\pi / \omega_{p i} \approx 500 \mathrm{~ns}$, during the initial rapid disorder-induced heating and oscillatory phase when the kinetic energy distribution as a function of temperature is not well known. Because this time scale varies with density, we expect different regions to equilibrate and oscillate at different times. Extracted ion temperatures should be viewed as more qualitative for these early times.

Equation (16) is the central result of this paper. It enables us to analyse the data in a clear and well-defined way, as shown in figure 8. It also separates the various contributions to the Doppler broadening of the spectrum. For example, figure $8(\mathrm{~A})$ shows the time dependence of $T_{i, \text { eff }}$ for a typical plasma. Figure $8(\mathrm{~B})$ shows the extracted $T_{i \text {,ave }}$ with the expansion effects removed. The only fit parameter in the model line in figure $8(\mathrm{~A})$ is $T_{i \text {, ave }}$. This procedure allows us to determine $T_{i \text {,ave }}$ to an accuracy of about $0.2 \mathrm{~K}$. Alternatively, after removing the effect of expansion by scaling the data by $0.95\left[1+(1 / 0.95)\left(t_{\text {delay }} / t_{\text {exp }}\right)^{2}\right]$, as in figure $8(\mathrm{~B})$, the equilibration temperature can be identified by the plateau. Scaling the data also improves the visibility of the disorder-induced heating during the first $1 / \omega_{p i} \approx 180 \mathrm{~ns}$. When there is significant electron heating $[7,9,8], T_{e}$ will exceed $3 E_{e} / 2 k_{\mathrm{B}}$, and $T_{e}$ then becomes a fit parameter that provides information on the electron dynamics. 

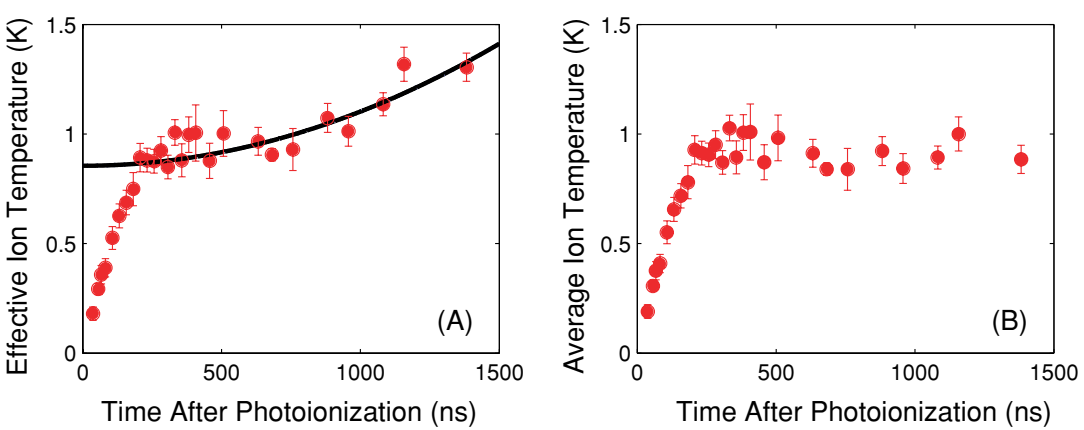

Figure 8. Evolution of the ion temperature in an ultracold neutral plasma. The initial peak ion density is $n_{0 i}=5 \times 10^{9} \mathrm{~cm}^{-3}$ and $\sigma_{i}=780 \mu \mathrm{m}$. The plasma contains $6 \times 10^{7}$ ions. (A) The effective ion temperature corresponding to the Doppler broadening extracted from Voigt profile fits of data such as in figure 4. The solid line (equation (16)) is the effective temperature one would expect for $T_{i \text {,ave }}=0.9 \mathrm{~K}$ and $T_{e}=2 E_{e} / 3 k_{\mathrm{B}}=40 \mathrm{~K}$. Equation (16) includes the effects of expansion but neglects early equilibration. (B) The average ion temperature is obtained by dividing by $0.95\left[1+(1 / 0.95)\left(t_{\text {delay }} / t_{\text {exp }}\right)^{2}\right]$.

Using this recipe for analysing the data we can conduct quantitative studies of the rate and equilibration temperature for disorder-induced heating. We can also examine the factors affecting ion temperature oscillations, electron temperature and plasma expansion. These will be the subjects of future work.

\section{Acknowledgments}

We thank A Chan, T Pohl, T Pattard and J M Rost for helpful discussion. This research was supported by the Department of Energy Office of Fusion Energy Sciences, National Science Foundation, Office for Naval Research, Research Corporation, Alfred P Sloan Foundation and David and Lucille Packard Foundation.

\section{References}

[1] Killian T C, Kulin S, Bergeson S D, Orozco L A, Orzel C and Rolston S L 1999 Phys. Rev. Lett. 834776

[2] Ichamuru S 1982 Rev. Mod. Phys. 541017

[3] Kulin S, Killian T C, Bergeson S D and Rolston S L 2000 Phys. Rev. Lett. 85318

[4] Killian T C, Lim M J, Kulin S, Dumke R, Bergeson S D and Rolston S L 2001 Phys. Rev. Lett. 863759

[5] Simien C E, Chen Y C, Gupta P, Laha S, Martinez Y N, Mickelson P G, Nagel S B and Killian T C 2004 Phys. Rev. Lett. 92143001

[6] Nagel S B, Simien C E, Laha S, Gupta P, Ashoka V S and Killian T C 2003 Phys. Rev. A 67011401

[7] Kuzmin S G and O’Neil T M 2002 Phys. Plasmas 93743

[8] Mazevet S, Collins L A and Kress J D 2002 Phys. Rev. Lett. 8855001

[9] Robicheaux F and Hanson J D 2003 Phys. Plasmas 102217

[10] Mitchell T B, Bollinger J J, Dubin D H E, Huang X-P, Itano W M and Baughman R H 1998 Science 2821290

[11] Mitchell T B, Bollinger J J, Huang X-P, Itano W M and Dubin D H E 1999 Phys. Plasmas 61751

[12] Bonitz M, Semkat D and Kremp D 1997 Phys. Rev. E 561246

[13] Zwicknagel G 1999 Contrib. Plasma Phys. 39155

[14] Morawetz K, Bonitz M, Morozov V G, Röpke G and Kremp D 2001 Phys. Rev. E 63020102

[15] Morozov I V and Norman G E 2003 J. Phys. A: Math. Gen. 366005

[16] Murillo M S 2001 Phys. Rev. Lett. 87115003

[17] Pohl T, Pattard T and Rost J M 2004 J. Phys. B: At. Mol. Opt. Phys. 37 L183

[18] Farouki R T and Hamaguchi S 1994 J. Chem. Phys. 1019885 
[19] Tkachev A N and Yakovlenko S I 2000 Quantum Electron. 301077

[20] Nicholson D R 1992 Introduction to Plasma Theory (Malabar, FL: Krieger)

[21] Dorozhkina D S and Semenov V E 1998 Phys. Rev. Lett. 812691

[22] Pohl T, Pattard T and Rost J M 2004 Phys. Rev. Lett. 92155003

[23] Siegman A E 1986 Lasers (Sausolito, CA: University Science Books)

[24] Hamaguchi S, Farouki R T and Dubin D H E 1997 Phys. Rev. E 564671

[25] Pohl T, Pattard T and Rost J M 2004 Private communication 